rial from the 10 of these patients who had undergone meningeal biopsy was also reviewed.

All 26 patients had postural headaches; complete alleviation was achieved by recumbency in 22 of them. Nausea or vomiting, neck pain, horizontal diplopia, changes in hearing, photophobia, upper limb pains or paresthesias, visual blurring, or dysgeusia was noted in some of the patients. Cardinal MRI features were diffuse pachymeningeal gadolinium enhancement $(100 \%)$, subdural collections of fluid $(69 \%)$, and evidence of descent of the brain $(62 \%)$ that sometimes resembled type I Chiari malformation. Only $46 \%$ of the patients had cerebrospinal fluid (CSF) opening pressures of $40 \mathrm{~mm}$ or less. In three patients, CSF pressures were consistently no less than 90 $\mathrm{mm} \mathrm{H} \mathrm{H}_{2} \mathrm{O}$ and as high as $130 \mathrm{~mm} \mathrm{H}_{2} \mathrm{O}$.

A variable pleocytosis of 5 or more cells per cubic millimeter was noted in 15 patients ( $>40$ cells $/ \mathrm{mm}^{3}$ in 4 patients). A variable increase in CSF protein was noted in at least one lumbar puncture in 23 patients. Six patients had overdraining CSF shunts; CSF leak was documented in another 11 patients. Shunt revision or ligation and surgical correction of the leak led to resolution of the clinical and MRI abnormalities in all cases thus treated. Four patients improved with eipidural blood patch. Three of the 12 patients treated supportively have remained symptomatic.

Histologically, a thin subdural zone of fibroblasts and thin-walled vessels was noted in an amorphous matrix. Two patients with prolonged symptoms had a more pronounced proliferative reaction.

The syndrome of low-pressure headaches and pachymeningeal gadolinium enhancement is being recognized with increasing frequency. The source of the CSF leak can be demonstrated in many patients. Meningeal abnormalities are likely attributable to decreased CSF volume and hydrostatic CSF pressure changes. The prognosis is typically good.

Mokri B, Piepgras DG, Miller GM: Syndrome of orthostatic headaches and diffuse pachymeningeal gadolinium enhancement. Mayo Clin Proc 1997;72:400-413.

\title{
Letters
}

\section{Musculoskeletal examination needs to be a matter of habit}

\section{To the Editor:}

Michael Patterson, $\mathrm{PhD}$, asks the question, "Why do not more osteopathic physicians in osteopathic medical hospitals perform the palpatory and structural examinations and look for somatic dysfunction as they have been trained to do?" (JAOA 1996;96:526). There are many reasons for this deficiency in hospital medical records that have been noted and reported over the past several decades by those of us who survey hospitals.

First, and very importantly, is the physician's failure to adequately document all the pertinent physical findings leading to a diagnosis and to adequately document absence of other physical findings that tend to rule out similar conditions. This failure is especially noted in outpatient surgery cases where written results from the physical may consist of only a few lines relative to the procedure to be done. Failure to document well is also seen in review of systems as well as family history. With regard to inpatient records, often times the nurse's written evaluations are more thorough than the physician's notes on the patient's history and physical.

Pursing this further, a musculoskeletal (MS) examination (such as that described in the Textbook of Physical Diagnosis, ed 2, Philadelphia, Pa, W.B. Saunders Co, 1994) and Documentation Guidelines for Evaluation and Management Services, Health Care Finance Administration, Dept of Health and Human Services, Washington, DC, 1994) should be done by both DOs and MDs as part of a complete physical examination. While allopathic physicians usually record an MS evaluation (posture, curvatures, gait, habitus, location of pain, and the like) many DOs seem to interpret an MS evaluation as being limited to osteopathic somatic dysfunction. Thus, they record the MS evaluation as being "negative," "not significant," or "not done because of the condition of the patient." Part of this problem can be attributed to the Accreditation Requirements for Acute Care Hospitals, issued by the American Osteopathic Association, which in former years, required evaluation of the spine in three positions of the patient. This required evaluation was so complex that it was difficult to perform on patients with acute problems. And although the required evaluation has been modified somewhat, it still fails to take into consideration that MS encompasses more than somatic dysfunction.

Another factor that has influenced the documentation (or more aptly the lack of documentation) of osteopathic somatic dysfunction is the trend of specialization by DOs. This specialization leads to increased focus on the disease and less on the patient, which frequently leads to a suspicion of specialist "tunnel vision." This tunnel vision often means that the physician overlooks somatic dysfunction as part of the patient's physical; the physician may fail to insist that interns or residents evaluate this area as well, thereby perpetuating such tunnel vision. Students who have spent 4 years learning osteopathic structural diagnosis and manipulative techniques now spend 4 years with no encouragement to use what they have learned. They see their mentors referring patients with spinal discomfort or pneumonia to physical therapy or respiratory therapy.

In this era of increasing awareness of alternative medicine, the osteopathic medical profession is in a very strong position to lead. I believe that many patients would benefit from and appreciate the value of the "laying on of hands." 
I suggest that students need to know that spinal somatic dysfunction may be a clue to the cause of vague or acute symptoms. The dysfunction may reflexly delay the healing of organic problems using medical therapy. Correcting the dysfunction will probably support and accelerate the healing response. In other cases, these dysfunctions may be incidental to the patient's chief complaint, but they may be of significance in the patient's long-term health.

Despite these points, many osteopathic hospitals fail to include the musculoskeletal examination as part of each patient's physical examination. In surveying a number of charts from osteopathic hospitals, I found in one large osteopathic teaching hospital 120 cases of chest pain diagnosed as "noncardiac" or "not otherwise specified." All these patients were admitted to the hospital through the emergency room and underwent thousands of dollars of tests. I evaluated 10 random charts of patients with these diagnoses. Not one patient had in his or her chart any record of musculoskeletal findings, such as pain in the ribs or sternum, spinal curves, or the like.

None of these records in this osteopathic hospital had included any history of the patient's lifestyle, possible trauma, or other symptoms present in other systems. Many patients were discharged with no suggestions given as to the cause of their dysfunction or were discharged without instructions for managing their condition. They were told they did not "have a heart attack."

(As a side note, I found one allopathic hospital in Michigan in which $50 \%$ of the patients' physical examinations included documentation of osteopathic somatic dysfunction by MDs. These findings were related to the chief complaint or incidental problems.)

Before the 1970s, it was routine for every patient in an osteopathic hospital to receive osteopathic manipulative treatment (OMT) at least once every day. Patients appreciated the OMT and often came to better understand their condition by talking with their physician about it while undergoing the OMT.

During the 1970s, it was decided that all therapies should be administered by order only; thus, routine manipulation was discontinued, and routine orders signed by the physician did not include OMT. The previously used spineogram was no longer acceptable, and spinal findings had to be written. This took longer and was more difficult. Because of this difficulty, many physicians recorded the patient's musculoskeletal findings as being "normal," "negative," or "not significant."

Similar problems were uncovered by Harry Friedman, DO, and his colleagues in their research (JAOA 1996;96:529536). It appears that the philosophy of osteopathic medicine and the value of and attention to musculoskeletal symptoms in the care of all patients must be reinforced at all levels of osteopathic medical training and practice, and it must be documented.

I offer the following suggestions. In the education of osteopathic medical students, interns, and residents, the importance of the osteopathic medical philosophy must be taught-and constantly reinforced-by those persons who understand and believe in it. The diagnosis of somatic lesions and the importance of proper manipulative management must be incorporated into all areas of teaching. So often, these students are taught the laboratory and imaging diagnostic techniques and pharmaceutical therapy on one day, and then during another class they are shown the musculoskeletal treatment component.

Fifty-five years ago, practicing DOs with an interest in the subject actually taught the clinical courses. These instructors all used and taught palpatory diagnosis and OMT every day for each of the body systems. Internships and residencies were evaluated using the same criteria for therapy.

The curriculum for graduate and postgraduate education, as well as the requirements for practicing in accredited institutions issued by the American Osteopathic Association (AOA), should be coordinated with the work being done at the specialty colleges, the American Association of Colleges of Osteopathic Medicine, the American Academy of
Osteopathy, and the Bureau of Healthcare Facilities Accreditation, among others. Together these groups should develop uniform guidelines that balance all aspects of diagnosis and care for patients.

\section{Martyn E. Richardson, DO}

Scarborough, Me

\section{Response}

\section{To the Editor:}

Martyn Richardson, DO, in his response to my editorial, suggests several interesting points. The lack of palpatory diagnosis and osteopathic manipulative treatment (OMT) records in hospital charts - despite the requirements for these records-is widely recognized. His analysis is important and insightful.

Perhaps chief among the reasons he cites are the lack of perceived importance and the perceived complexity of the process. Are we making the teaching of osteopathic medical diagnosis and OMT so complex that students are perceiving it as a specialty that they cannot really do? Are we failing to communicate the true importance of the somatic component of health (and disease)? If osteopathic physicians perceived the importance of musculoskeletal function and dysfunction in all aspects of health and disease, there would be much more use made of the palpatory, diagnostic, and treatment modalities, especially if recording the findings could be done easily.

Dr Richardson addreses these points, and in a disquieting comment, notes that in one allopathic hospital extensive use is made of musculoskeletal findings. This use is encouraging in terms of an acceptance of the importance of such findings in the treatment of patients. But it raises questions regarding the importance placed on musculoskeletal findings in osteopathic medical institutions. Perhaps the accrediting bodies of the American Osteopathic Association need to be more attendant to the requirements for musculoskeletal examination.

The osteopathic medical profession has a heritage of clinical use of muscu- 